\title{
Very Good
}

National Cancer Institute

\section{Source}

National Cancer Institute. Very Good. NCI Thesaurus. Code C103342.

An indication that something has a high deg ree of quality, value or worth. 